\title{
Regeneración de plantas leñosas en el Chaco semiárido argentino: relación con factores bióticos y abióticos en micrositios creados por el aprovechamiento forestal
}

\author{
Woody plant regeneration in the semiarid Chaco forest: relationship with biotic and abiotic \\ factors in microsites created by logging
}

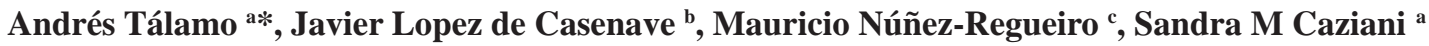 \\ *Autor de correspondencia: ${ }^{a}$ Instituto de Bio y Geociencias del Noroeste Argentino (IBIGEO), Universidad Nacional de Salta \\ (UNSa) y Consejo Nacional de Investigaciones Científicas y Técnicas (CONICET), Mendoza 2, Salta, Argentina, \\ tel.: 54-387-154 448066, atalamo@unsa.edu.ar

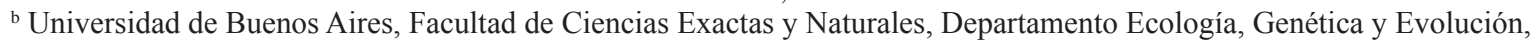 \\ Buenos Aires, Argentina. \\ ${ }^{\mathrm{c}}$ University of Florida, Wildlife Ecology and Conservation Department, Florida, USA.
}

\begin{abstract}
SUMMARY
Logging activities generate microsites that can be colonized by different woody plant species whose regeneration may be influenced by biotic and abiotic factors. This work describes three microsites created by logging (main roads, skid trails and logging gaps) in two sites with different recovery time (one and two years) in the semi-arid Chaco of Argentina. Woody plant richness and diversity are compared and the relationship of plant density as a function of microsite type, recovery time, soil compaction, canopy cover and herbaceous cover is evaluated. Richness and species composition were similar in all microsites, which were dominated by the same species. Main roads presented more compacted soil, skid trails had denser canopies and logging gaps had soils with larger herbaceous cover. Woody plant density depended on the interaction of the covariates (soil compaction, canopy cover and herbaceous cover) with microsites and recovery time. Road plant density was low and was negatively related with soil compaction and positively related with canopy cover as well as with herbaceous cover, suggesting a positive interaction (facilitation) in microsites that were most affected by logging. The regeneration of the two most valuable timber species was low compared to other species. In the microsites studied, Aspidosperma quebracho-blanco had a similar density and Schinopsis lorentzii a higher density compared to the regeneration found in the surrounding matrix reported in other studies, but this result should be interpreted with caution due to the low constancy (proportion of plot containing a certain taxon). We propose, cautiously, that generated microsites would not be negatively affecting the regeneration of the two most valuable timber species in this environment.
\end{abstract}

Key words: logging roads, gaps, canopy cover, soil compaction, skid trails.

\section{RESUMEN}

La cosecha forestal genera micrositios que pueden ser colonizados por distintas especies de plantas leñosas, donde factores bióticos y abióticos pueden influir en la regeneración. En este trabajo se describen tres micrositios creados por la tala (caminos principales, vías de saca y claros de extracción) en dos sitios con distinto tiempo de recuperación (uno y dos años) del Chaco semiárido, Argentina. Se compara la riqueza y diversidad de plantas leñosas y se evalúa la relación de la densidad de plantas en función del tipo de micrositio, tiempo de recuperación, dureza del suelo, cobertura del dosel y cobertura de herbáceas. En los tres micrositios, la composición y riqueza de especies fueron similares, dominados por las mismas especies. Los caminos tuvieron suelos más compactados, las vías de saca doseles más cerrados y los claros suelos con mayor cobertura herbácea. La densidad de leñosas dependió de la interacción de las co-variables (dureza del suelo, coberturas del dosel y de herbáceas) con los micrositios y el tiempo de recuperación. La densidad en los caminos fue baja y se relacionó negativamente con la dureza del suelo y positivamente con las coberturas del dosel y de herbáceas, sugiriendo una interacción positiva en los micrositios más afectados. La regeneración de las dos especies de mayor valor forestal fue baja en comparación a otras especies; Aspidosperma quebracho-blanco tuvo densidad similar y Schinopsis lorentzii mayor en comparación a la regeneración de la matriz circundante. Sin embargo, este resultado debe interpretarse con cuidado debido a la baja constancia de ambas especies (proporción de parcelas con un cierto taxón). Se propone, con cautela, que los micrositios generados no estarían afectando negativamente a la regeneración de las dos especies de mayor valor forestal de este ambiente.

Palabras clave: caminos, claros, cobertura del dosel, dureza del suelo, vías de saca. 


\section{INTRODUCCIÓN}

Un proceso clave para garantizar la sustentabilidad ecológica de la actividad forestal es la regeneración del bosque, que depende de múltiples factores (bióticos y abióticos) cuya interacción definirá sus efectos netos sobre el sistema (Mostacedo y Frederiksen 2001, Grulke et al. 2007, PeñaClaros et al. 2008). Estudiar la regeneración natural en bosques aprovechados forestalmente y comprender qué mecanismos operan es un gran desafío para los profesionales del manejo y la conservación de los recursos naturales. Una de las consecuencias de la actividad forestal es la creación de nuevos micrositios, como por ejemplo caminos, vías de saca de distinto orden, claros de extracción y pistas en donde se acumulan los productos forestales, entre otros. En estos nuevos micrositios se modifica la disponibilidad de recursos (luz, espacio, nutrientes) y, una vez que se abandona la actividad forestal, son sitios potencialmente aptos para el reclutamiento de individuos de especies leñosas maderables y no maderables (Guariguata y Dupuy 1997, Park et al. 2005, Nabe-Nielsen et al. 2007, Peña-Claros et al. 2008), más aún si se utilizan técnicas de tala de impacto reducido (Pinard et al. 2000).

El éxito de la regeneración de las plantas leñosas en los micrositios creados por la actividad forestal podría depender de factores como las condiciones físicas del suelo (dureza, infiltración, profundidad del mantillo), el porcentaje de luz incidente, la competencia de las plántulas con gramíneas y herbáceas dicotiledóneas, y el tiempo transcurrido desde el cese de la explotación forestal, entre otros (Nicotra et al. 1999, Park et al. 2005, Nabe-Nielsen et al. 2007, Anthelme y Michalet 2009, Toledo-Aceves et al. 2009, Ampoorter et al. 2011). Bajo cualquier sistema de aprovechamiento forestal, el suelo es modificado de diferentes maneras e intensidades y la compactación del suelo podría tener importantes efectos sobre la regeneración del bosque. Sin embargo, estos efectos no siempre son negativos, ya que dependiendo del tipo de bosque y del tipo de suelo, los efectos pueden ser despreciables y hasta levemente positivos (Ampoorter et al. 2011). El ambiente lumínico también es alterado en los bosques bajo aprovechamiento forestal, lo cual a su vez puede modificar la comunidad de renovales (Nicotra et al. 1999, Nabe-Nielsen et al. 2007). En bosques tropicales de Bolivia se han identificado especies que se benefician por la mayor intensidad de luz en los caminos abandonados (Nabe-Nielsen et al. 2007) o en los grandes claros de extracción (Park et al. 2005). Pero las plántulas y renovales de las especies leñosas no crecen aisladas, sino que interactúan con gramíneas y herbáceas dicotiledóneas, y el efecto de dicha interacción podría ser tanto negativo (competencia) como positivo (facilitación) (Davis et al. 1998, Anthelme y Michalet 2009). Una vez que el aprovechamiento forestal es abandonado, el tiempo transcurrido desde el abandono también puede influir en los patrones de regeneración (Toledo-Aceves et al. 2009). En bosques tropicales de Bolivia, la composición de los ensambles de especies pioneras (plántulas y juveniles) y sus densidades cambiaron con el tiempo de abandono de los sitios estudiados, aunque no así las especies de interés comercial (Park et al. 2005).

El Chaco semiárido se encuentra bajo diferentes presiones que ponen en riesgo su subsistencia en el futuro (Zak et al. 2004). A pesar de que uno de los usos de la tierra más comunes, en el pasado y en la actualidad, es la extracción de maderas duras, el aprovechamiento forestal se realiza sin ningún ordenamiento espacial ni temporal y sin tener en cuenta la regeneración de las especies forestalmente importantes (Brassiolo et al. 2001, Grulke et al. 2007). Generalmente, el sistema empleado es el de selección de árboles individuales o "cortas por huroneo", extrayéndose solamente los individuos de mejor calidad y dejando un bosque empobrecido desde el punto de vista forestal. En un lote aprovechado por este sistema es común encontrar (1) caminos principales, (2) caminos secundarios o vías de saca y (3) espacios abiertos (claros) originados por la extracción de los árboles individuales (Grulke et al. 2007). Los caminos principales se utilizan para la movilización de los productos forestales mediante camiones o tractores con acoplado, por lo que son sectores abiertos (4-6 m de ancho), con suelos compactados y baja cobertura vegetal. Las vías de saca son senderos construidos a machete por donde el rollo o poste se extrae manualmente o mediante "zorras" (carros tirados por mulas), por lo que son sectores más cerrados (1-2 m de ancho), con mayor cobertura del dosel, con el suelo menos compactado y con mayor cobertura vegetal. Por último, los claros son pequeños espacios abiertos (4-7 $\mathrm{m}$ de diámetro) que se generan al apear el árbol blanco, quedando un sector relativamente abierto, con vegetación sobreviviente y con el suelo poco compactado en comparación a los caminos (Tálamo 2006, Grulke et al. 2007). A pesar de que estos micrositios están muy extendidos en el Chaco semiárido, no existen estudios que evalúen la regeneración natural en los mismos.

Dado el escaso conocimiento sobre la regeneración natural de plantas leñosas en ambientes modificados en el Chaco semiárido y reconociendo la importancia de comprender este proceso clave en un sistema boscoso amenazado, los objetivos de este trabajo son: (1) describir la dureza del suelo, la cobertura del dosel y la cobertura de plantas herbáceas de los tres micrositios (caminos principales, vías de saca de tercer orden y claros de extracción) en dos sitios con diferente tiempo de recuperación desde el abandono de la actividad forestal, (2) comparar la riqueza y diversidad de la comunidad de plantas leñosas en los tres micrositios de ambos sitios, y (3) evaluar la relación entre la densidad de plantas leñosas y las tres variables mencionadas en el objetivo (1), en función del tipo de micrositio y del tiempo de recuperación.

Si las características de los distintos micrositios resultan diferentes, tal como se espera, y los factores bióticos y abióticos evaluados influyen en los patrones de regeneración, se predice que (1) la composición de especies del en- 
samble de plantas leñosas será diferente en micrositios con distinto grado de cobertura de dosel y (2) la densidad de plantas leñosas será menor en micrositios con suelos más compactados y con mayor cobertura de plantas herbáceas.

\section{MÉTODOS}

Área de estudio. El trabajo fue realizado en dos sitios que fueron aprovechados mediante cortas por huroneo: sitio 1 y sitio 2, explotados uno y dos años antes de la toma de datos, respectivamente. Ambos sitios se encuentran ubicados en la provincia de Chaco, al norte y al este del Parque Nacional Copo $25^{\circ} 55^{\prime} \mathrm{S} 62^{\circ} 05^{\prime} \mathrm{O}$ (figura 1). El paisaje es plano y está compuesto por un mosaico de bosques semideciduos y espinosos interrumpido por franjas de pastizales naturales asociados a paleocauces (Morello y Adámoli 1974). El clima es marcadamente estacional, con el $80 \%$ de las precipitaciones anuales $(700 \mathrm{~mm})$ concentradas entre octubre y marzo (primavera-verano). Una descripción más detallada de la vegetación leñosa del área de estudio se encuentra en Tálamo (2006).

Diseño del estudio. En cada sitio se seleccionaron réplicas de cada tipo de micrositio (camino, vía de saca y claro) y dentro de cada una de estas réplicas se midieron las variables de respuesta y las co-variables en parcelas de $1 \mathrm{x}$ $1 \mathrm{~m}$. En los claros se dispusieron siempre cuatro parcelas ubicadas al norte, sur, este y oeste del centro del claro. En las vías de saca y en los caminos principales, el número de parcelas estuvo sujeto a la longitud de cada caso (cuadro 1, figura 1). Cada parcela estuvo distanciada de las otras aproximadamente por unos $15 \mathrm{~m}$. Las variables de

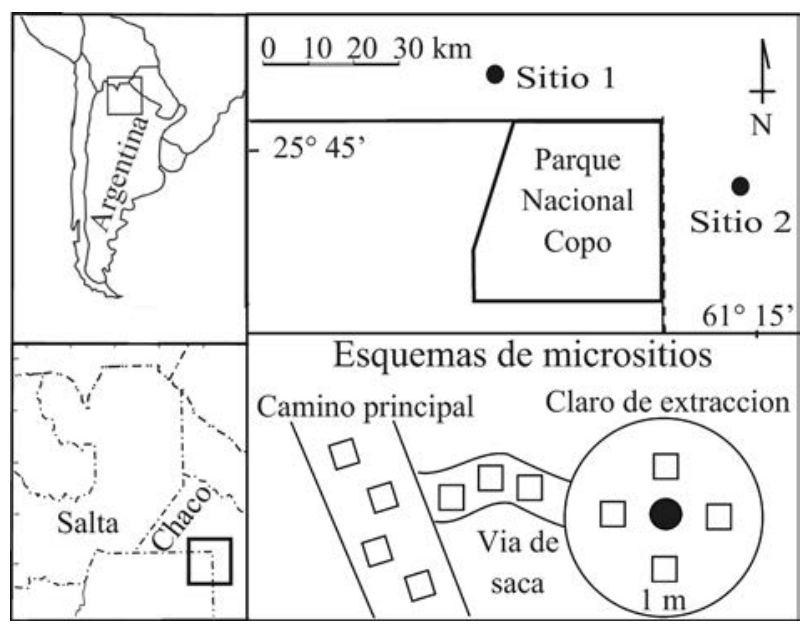

Figura 1.Ubicación de los dos sitios (sitio 1 y sitio 2) aprovechados forestalmente en el bosque chaqueño semiárido de los alrededores del Parque Nacional Copo, Argentina. Se muestra además un esquema de los micrositios y del diseño de muestreo.

Locations of studied sites (site 1 and site 2) with selective logging in the semiarid Chaco forest near Copo National Park, Argentina, and scheme of microsites and sampling design used. respuesta registradas en cada parcela fueron la densidad de plantas leñosas (número de individuos por parcela) y el número de especies por parcela, mientras que las covariables fueron la dureza del suelo, medida usando un penetrómetro de bolsillo (Forestry Suppliers Pocket Penetrometer) y registrando cinco mediciones por parcela, la cobertura del dosel, usando un densímetro esférico convexo (Forestry Suppliers Spherical Crown Densiometer) y registrando cuatro mediciones por parcela y la superficie del suelo cubierto por plantas herbáceas (en porcentaje, estimado visualmente).

Análisis de datos. Para el objetivo 1, las variables dureza del suelo (de ahora en más llamada "dureza"), cobertura del dosel (de ahora en más llamada "dosel") y cobertura de herbáceas (de ahora en más llamada "herbáceas") fueron resumidas mediante gráficos de cajas y analizadas de manera independiente mediante un Análisis de la Varianza, previa verificación de los supuestos mediante un análisis de residuales (Quinn y Keough 2002).

Con respecto al objetivo 2 , debido a que la riqueza de especies observada puede depender del esfuerzo de muestreo (número de parcelas), las comparaciones se realizaron mediante un análisis de rarefacción basado en muestras (Colwell et al. 2012). El análisis se realizó usando el programa EstimateS (Versión 8.2.0) (Colwell 2011), el cual calcula la riqueza promedio de muestras de parcelas creciente, con 99 repeticiones para cada nivel de parcela. Además, se calculó el intervalo de confianza del $95 \%$ para un mismo número de parcelas para comparar la riqueza de especies promedio entre micrositios. La diversidad de especies se analizó mediante curvas de rango-abundancia, las cuales permiten comparar gráficamente la riqueza de especies (número de puntos), sus abundancias relativas, la forma de las curvas (equitatividad) y la secuencia de cada una de las especies que componen la comunidad sin perder su identidad (Feinsinger 2004).

Cuadro 1. Esfuerzo de muestreo utilizado en tres micrositios creados por la actividad forestal selectiva en sitios con un año (sitio 1) y dos años de recuperación (sitio 2) en el bosque chaqueño semiárido de los alrededores del Parque Nacional Copo, Argentina.

Sampling effort used in three microsites created by selective logging in sites with recovery time of one year (site 1) and two years (site 2) in the semiarid Chaco forest near Copo National Park, Argentina.

\begin{tabular}{clccc}
\hline \multirow{2}{*}{ Sitio } & \multirow{2}{*}{ Micrositio } & \multirow{2}{*}{ Réplicas } & \multicolumn{2}{c}{ Parcelas de 1 x $1 \mathrm{~m}$} \\
\cline { 4 - 5 } & & & Por réplica & Total \\
\hline \multirow{3}{*}{ Sitio 1 } & Caminos & 3 & $4-14$ & 29 \\
& Vías de saca & 7 & $1-5$ & 20 \\
& Claros & 7 & 4 & 28 \\
\hline \multirow{2}{*}{ Sitio 2 } & Caminos & 4 & 5 & 20 \\
& Vías de saca & 8 & $1-3$ & 17 \\
& Claros & 8 & 4 & 32 \\
\hline
\end{tabular}


Para el objetivo 3, la asociación entre la densidad de plantas leñosas y las variables explicativas (sitio, micrositio, dureza, dosel, herbáceas y las interacciones) fue analizada construyendo modelos lineales generalizados en el programa R (R Development Core Team 2010) usando la función "glm" (que utiliza para la estimación de parámetros el método de máxima verosimilitud). $\mathrm{Al}$ ser los valores de la variable de respuesta números positivos que surgen de conteos, se creó un modelo siguiendo una distribución Poisson y una función de enlace del tipo Log. Primero se ajustó un modelo con los factores sitio, micrositio y su interacción, luego se construyó un modelo completo agregando al modelo inicial los términos de los efectos de las covariables (dureza, dosel, herbáceas y las interacciones con los factores sito y micrositio) y este modelo completo fue simplificado eliminando los efectos que no fueron significativos. La selección de modelos se realizó comparando valores obtenidos a partir del criterio de información de Akaike (AIC, Burnham y Anderson 2002). Para mostrar la relación entre las co-variables y la densidad de plantas leñosas, así como sus interacciones con los factores sitio y micrositio, se realizaron gráficos de dispersión con curvas de ajuste suavizadas (para obtener una representación gráfica más clara) mediante la función Lowess usando el programa R.

\section{RESULTADOS}

Descripción de los micrositios. La dureza del suelo resultó mayor en los caminos en comparación a las vías y los claros y, en promedio, el sitio 2 tuvo suelos más duros, resultando ambos factores independientes (figura $2 \mathrm{~A}$ y cuadro 2). La cobertura del dosel fue mayor en las vías y en el sitio 1 , mientras que el efecto de los micrositios dependió del sitio considerado (figura $2 \mathrm{~B}$ y cuadro 2). Por último, la cobertura de plantas herbáceas fue mayor en los claros, intermedia en las vías y baja en los caminos y, en promedio, fue mayor en el sitio 2 , siendo ambos factores independientes entre sí (figura 2C y cuadro 2).

Riqueza y diversidad de especies. Comparando los tres micrositios a un mismo esfuerzo de muestreo (17 parcelas), no se puede afirmar que la riqueza promedio de especies sea diferente en los micrositios de ambos sitios estudiados, dado que los intervalos de confianza para la riqueza estimada de cada micrositio se superponen ampliamente (cuadro 3).

Los tres micrositios estuvieron dominados por las mismas especies (Celtis pallida Torr y Acacia praecox Griseb.) en los dos sitios estudiados (figura 3). En el sitio 1 los ensambles de plantas fueron menos equitativos debido a la alta dominancia de Acacia praecox (en los caminos y las vías de saca) y de C. pallida y Acacia praecox (en los claros). En el sitio 2, con un año más de recuperación, los ensambles fueron más equitativos; Acacia praecox dominó en los claros, C. pallida en los caminos y ambas especies en las vías de saca. Las especies de mayor importancia forestal (Aspi-

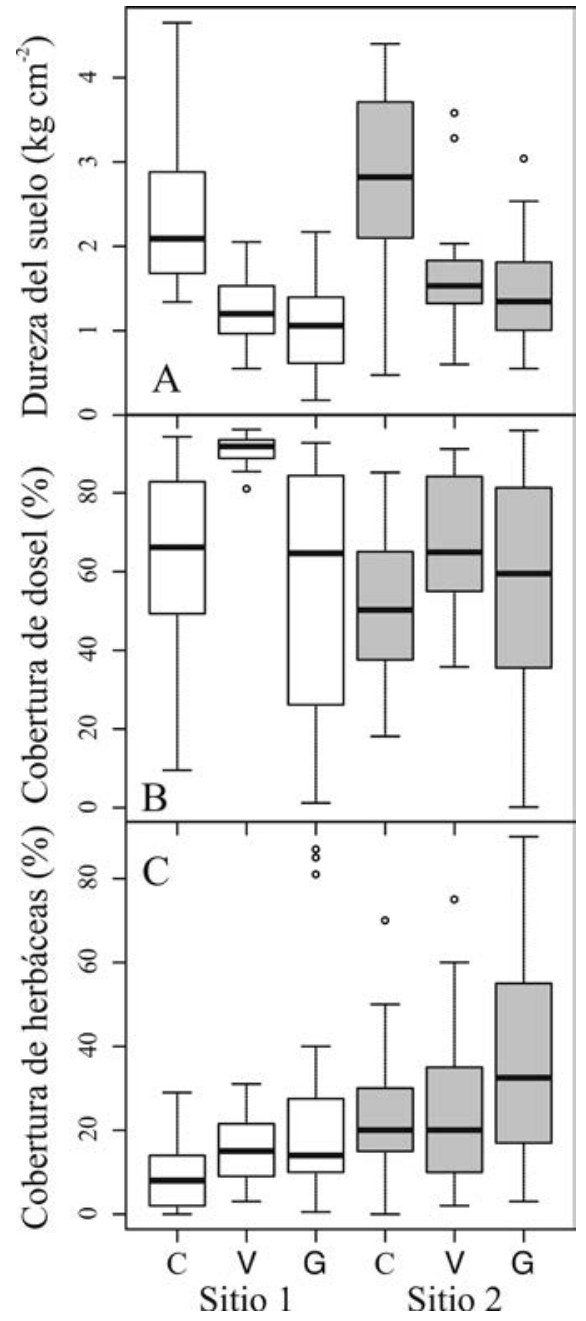

Figura 2.Dureza del suelo (A), cobertura del dosel (B) y cobertura de plantas herbáceas $(\mathrm{C})$ en tres micrositios creados por la actividad forestal selectiva (C: caminos principales, $\mathrm{V}$ : vías de saca, G: claros de extracción) en sitios con un año (sitio 1) y dos años de recuperación (sitio 2) en el bosque chaqueño semiárido de los alrededores del Parque Nacional Copo, Argentina.

Soil compaction (A), canopy cover (B) and herbaceous cover (C) in three microsites created by selective logging (C: main roads, $\mathrm{V}$ : skid trails and G: logging gaps) in sites with recovery time of one year (site 1) and two years (site 2) in the semiarid Chaco forest near Copo National Park, Argentina.

dosperma quebracho-blanco Schlecht. y Schinopsis lorentzii (Griseb.) Engl.) estuvieron representadas en muy bajas densidades y con mucha dispersión (cuadro 4 y figura 3).

Densidad de individuos en función de los factores y las covariables. En términos generales, la densidad de plantas leñosas fue menor en los caminos principales en comparación a las vías de saca y a los claros de extracción (cuadro 4).

$\mathrm{Al}$ ajustar los dos primeros modelos teniendo en cuenta los factores micrositio, sitio y su interacción, se encontró según el modelo 2 (cuadro 5), que existió un efecto alta- 
Cuadro 2. Resultados del análisis de la varianza para las variables dureza del suelo, cobertura del dosel y cobertura de plantas herbáceas. Analysis of variance results for soil compaction, canopy cover and herbaceous cover.

\begin{tabular}{llccc}
\hline \multirow{2}{*}{ Variables } & \multicolumn{1}{c}{ Fuente de variación } & g.l. & Parámetros \\
\cline { 3 - 4 } Dureza del suelo & Sitios & 2 & 44,53 & \multicolumn{1}{c}{ F } \\
& Micrositios & 1 & 7,03 & 0,0001 \\
& Sitios x Micrositios & 2 & 0,18 & 0,83 \\
\hline \multirow{3}{*}{ Cobertura del dosel } & Sitios & 2 & 12,6 & $<0,0001$ \\
& Micrositios & 1 & 7,64 & 0,006 \\
& Sitios x Micrositios & 2 & 3,6 & 0,03 \\
\hline \multirow{3}{*}{ Cobertura herbáceas } & Sitios & 2 & 8,64 & 0,0002 \\
& Micrositios & 1 & 17,84 & $<0,0001$ \\
& Sitios x Micrositios & 2 & 0,35 & 0,7 \\
\hline
\end{tabular}

Cuadro 3. Riqueza promedio de especies de plantas leñosas (S) estimada para un mismo esfuerzo de muestreo (17 parcelas) en tres micrositios creados por la actividad forestal selectiva (C: caminos principales, V: vías de saca, G: claros de extracción) en sitios con un año (sitio 1) y dos años de recuperación (sitio 2) en el bosque chaqueño semiárido de los alrededores del Parque Nacional Copo, Argentina. IC: intervalo de confianza.

Average richness of woody plant species estimated at a constant sampling effort (17 plots) in three microsites created by selective logging (C: main roads, V: skid trails and G: logging gaps) in sites with recovery time of one year (site 1) and two years (site 2) in the semiarid Chaco forest near Copo National Park, Argentina. IC = confidence interval.

\begin{tabular}{cccc}
\hline \multirow{2}{*}{ Parámetro } & $\mathrm{C}$ & $\mathrm{V}$ & $\mathrm{G}$ \\
\cline { 2 - 4 } & & Sitio 1 & 7 \\
$\mathrm{~S}_{(17 \text { parcelas })}$ & 5,9 & 9,2 & {$[3,3-10,6]$} \\
$\mathrm{IC}(95 \%)$ & {$[2,5-9,2]$} & {$[4,7-13,8]$} & \\
\hline & & Sitio 2 & 9,8 \\
\hline $\mathrm{S}_{(17 \text { parcelas })}$ & 11 & 11 & {$[5,9-13,6]$} \\
IC $(95 \%)$ & {$[7,2-14,9]$} & {$[5,7-16,3]$} & \\
\hline
\end{tabular}

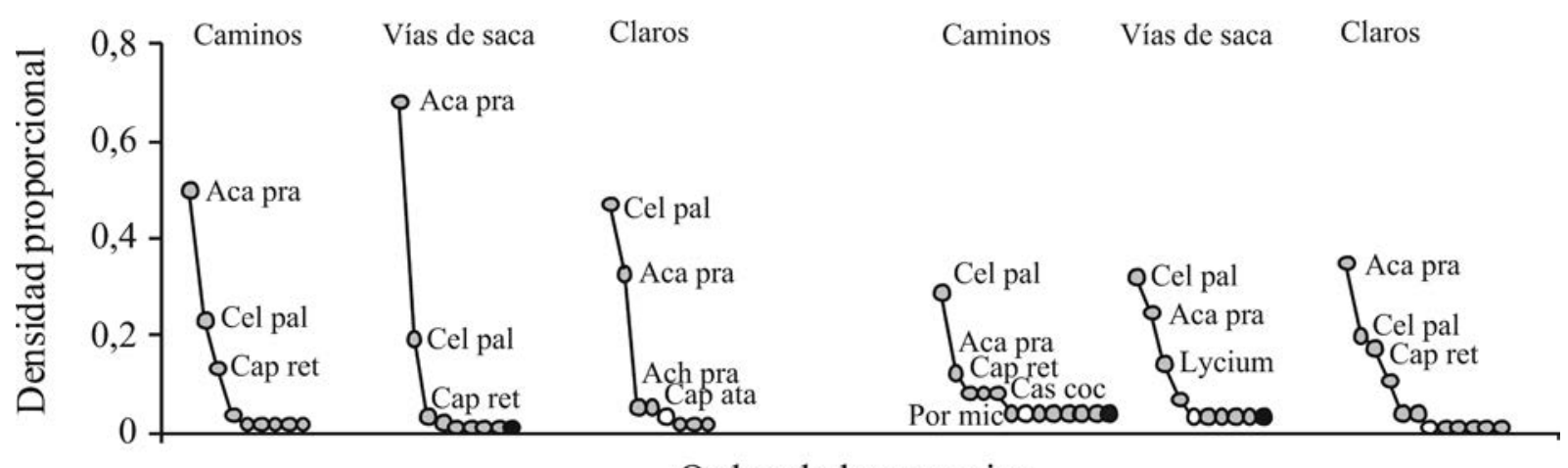

Sitio 1

Sitio 2

Figura 3. Curvas de rango-abundancia de las especies de plantas leñosas presentes en tres micrositios creados por la actividad forestal selectiva en sitios con un año (sitio 1) y dos años de recuperación (sitio 2) en el bosque chaqueño semiárido de los alrededores del Parque Nacional Copo, Argentina. Cel pal: Celtis pallida, Aca pra: Acacia praecox, Cap ret: Capparis retusa Griseb., Cas coc: Castela coccinea Griseb., Por mic: Porlieria microphylla (Baill.) Descole, Ach pra: Achatocarpus praecox Griseb., Cap ata: Capparis atamisquea Kuntze, círculo blanco: Aspidosperma quebracho-blanco, círculo negro: Schinopsis lorentzii.

Rank abundance curves of woody plant species present in three microsites created by selective logging in sites with recovery time of one year (site 1) and two years (site 2) in the semiarid Chaco forest near Copo National Park, Argentina. Cel pal: Celtis pallida, Aca pra: Acacia praecox, Cap ret: Capparis retusa Griseb., Cas coc: Castela coccinea Griseb., Por mic: Porlieria microphylla (Baill.) Descole, Ach pra: Achatocarpus praecox Griseb., Cap ata: Capparis atamisquea Kuntze, white circle: Aspidosperma quebracho-blanco, black circle: Schinopsis lorentzii. 
Cuadro 4. Medidas descriptivas de la densidad de plantas leñosas (número de individuos $\mathrm{m}^{-2}$ ) encontrada en tres micrositios creados por la actividad forestal selectiva (C: caminos principales, V: vías de saca, G: claros de extracción) en sitios con un año (sitio 1) y dos años de recuperación (sitio 2) en el bosque chaqueño semiárido de los alrededores del Parque Nacional Copo, Argentina. Se indica la densidad total y de las especies de interés forestal. DS: desvío estándar, Q1: primer cuartil, Q3: tercer cuartil.

Descriptive measures of woody plant density (number of individuals per $\left.\mathrm{m}^{2}\right)$ found in three microsites created by selective logging $(\mathrm{C}$ : main roads, V: skid trails and G: logging gaps) in sites with recovery time of one year (site 1) and two years (site 2) in the semiarid Chaco forest near Copo National Park, Argentina. DS: standard deviation, Q1 = first quartile, Q3 = third quartile.

\begin{tabular}{|c|c|c|c|c|c|c|c|}
\hline Sitio & Micrositio & Densidad & Promedio & DS & Q1 & Mediana & Q3 \\
\hline \multirow{9}{*}{ Sitio 1} & \multirow{3}{*}{$\mathrm{C}$} & Total & 1,9 & 2,5 & 0 & 1 & 3 \\
\hline & & Schinopsis lorentzii & 0 & 0 & 0 & 0 & 0 \\
\hline & & Aspidosperma quebracho-blanco & 0 & 0 & 0 & 0 & 0 \\
\hline & \multirow{3}{*}{$\mathrm{V}$} & Total & 4,5 & 6,7 & 1 & 3 & 5 \\
\hline & & Schinopsis lorentzii & 0,05 & 0,22 & 0 & 0 & 0 \\
\hline & & Aspidosperma quebracho-blanco & 0 & 0 & 0 & 0 & 0 \\
\hline & \multirow{3}{*}{ G } & Total & 2,3 & 2,1 & 1 & 1,5 & 4 \\
\hline & & Schinopsis lorentzii & 0 & 0 & 0 & 0 & 0 \\
\hline & & Aspidosperma quebracho-blanco & 0,07 & 0,26 & 0 & 0 & 0 \\
\hline \multirow{9}{*}{ Sitio 2} & \multirow{3}{*}{$\mathrm{C}$} & Total & 1,4 & 1,6 & 0 & 1 & 2 \\
\hline & & Schinopsis lorentzii & 0,05 & 0,22 & 0 & 0 & 0 \\
\hline & & Aspidosperma quebracho-blanco & 0,05 & 0,22 & 0 & 0 & 0 \\
\hline & \multirow{3}{*}{$\mathrm{V}$} & Total & 3 & 2 & 2 & 2 & 4 \\
\hline & & Schinopsis lorentzii & 0,06 & 0,24 & 0 & 0 & 0 \\
\hline & & Aspidosperma quebracho-blanco & 0,06 & 0,24 & 0 & 0 & 0 \\
\hline & \multirow{3}{*}{ G } & Total & 3,7 & 2,1 & 2 & 3 & 5 \\
\hline & & Schinopsis lorentzii & 0 & 0 & 0 & 0 & 0 \\
\hline & & Aspidosperma quebracho-blanco & 0,03 & 0,18 & 0 & 0 & 0 \\
\hline
\end{tabular}

Cuadro 5. Modelos evaluados y sus respectivos AIC para la variable de respuesta densidad de individuos (número de individuos por parcela). $\mathrm{M}=$ micrositio, $\mathrm{S}=$ sitio, Dur = dureza, Dos $=$ dosel y Herb = herbáceas.

Used models and their respective AIC for the response variable woody plant density (number of individuals per $\mathrm{m}^{2}$ ). $\mathrm{M}=$ microsite, $\mathrm{S}=$ site, Dur $=$ soil compaction, Dos $=$ canopy cover, and Herb $=$ herbaceous cover.

\begin{tabular}{clc}
\hline $\mathrm{N}^{\circ}$ & \multicolumn{1}{c}{ Modelo } & AIC \\
\hline 1 & $\mathrm{M}+\mathrm{S}+\mathrm{M} * \mathrm{~S}$ & 728,76 \\
2 & $\mathrm{M}+\mathrm{S}$ & 727,86 \\
3 & $\mathrm{M}+\mathrm{S}+\mathrm{M} * \mathrm{~S}+$ Dur + Dos + Herb + Dur*S + Dur*M + Dos*S + Dos*M + Herb*S + Herb*M & 697,53 \\
4 & $\mathrm{M}+\mathrm{S}+\mathrm{M} * \mathrm{~S}+$ Dur + Dos + Herb + Dur*S + Dur*M + Dos*S + Herb*S + Herb*M & 695,53 \\
5 & $\mathrm{M}+\mathrm{S}+$ Dur + Dos + Herb + Dur*S + Dur*M + Dos*S + Herb*S + Herb*M & 693,94 \\
6 & $\mathrm{M}+\mathrm{S}+$ Dur + Dos + Herb + Dur*S + Dur*M + Herb*S + Herb*M & 694,22 \\
7 & $\mathrm{M}+\mathrm{S}+$ Dur + Dos + Herb + Dur*M + Herb*S + Herb*M & 694,40 \\
\hline
\end{tabular}

mente significativo del factor micrositio (parámetro estimado $=-0,37608 ; \mathrm{EE}=0,06547 ; t=-5,745$ y $P=9,21$ x $10^{-09}$ ) y una falta de efecto del factor sitio (parámetro estimado $=0,03477 ; \mathrm{EE}=0,09920 ; t=0,350$ y $P=0,726$ ). Luego, al trabajar con el modelo completo (agregando todas las co-variables y sus interacciones con ambos factores), el ajuste mejoró considerablemente (modelo 3; cuadro 5). Dicho modelo completo fue simplificado, eli- minando distintos efectos hasta llegar al mejor modelo que presentó el menor AIC (modelo 5; cuadro 5).

Interpretando el modelo 5 , se puede proponer que la densidad de plantas leñosas se relacionó significativamente con las tres co-variables incluidas (dosel, dureza y herbáceas). El efecto de la dureza dependió del factor micrositio y tendió a depender del factor sitio (cuadro 6 y figura 4): en suelos muy blandos la densidad de plantas 


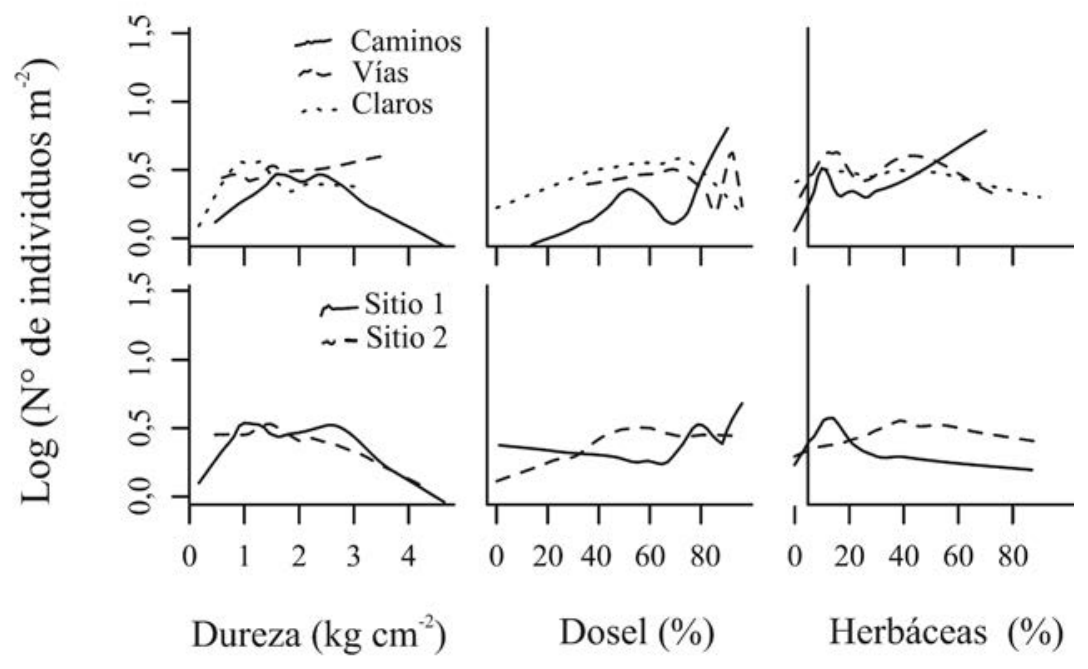

Figura 4. Relación entre la densidad de individuos de plantas leñosas (expresado logarítmicamente) y las co-variables dureza del suelo, cobertura del dosel y cobertura de plantas herbáceas en tres micrositios creados por la actividad forestal selectiva en sitios con un año (sitio 1) y dos años de recuperación (sitio 2) en el bosque chaqueño semiárido de los alrededores del Parque Nacional Copo, Argentina. Se muestran las curvas suavizadas sin las nubes de puntos para una visualización más clara de las tendencias.

Relationship between woody plant density (logarithmic scale) and covariates soil compaction, canopy cover and herbaceous cover in three microsites created by selective logging (main roads, skid trails and logging gaps) in sites with recovery time of one year (site 1) and two years (site 2) in the semiarid Chaco forest near Copo National Park, Argentina. Smoothed curves are shown for a clear interpretation of tendencies.

Cuadro 6. Parámetros estimados, errores estándar (EE), valores de $t$ y valores de $P$ correspondientes al modelo 5 (ver cuadro 5 ) con menor AIC para la variable de respuesta densidad de individuos (número de individuos por parcela).

Estimated parameters, standard errors, $t$ values and $P$ values for model 5 with the lowest AIC for the response variable woody plant density (number of individuals $\mathrm{m}^{-2}$ ).

\begin{tabular}{lcccc}
\hline Parámetro & Valor & EE & $t$ & $P$ \\
\hline Micrositio & $-0,145967$ & 0,176199 & $-0,828$ & 0,40743 \\
Sitio & 0,569729 & 0,412952 & 1,380 & 0,16769 \\
Dureza & 0,529641 & 0,247572 & 2,139 & 0,03241 \\
Dosel & 0,009259 & 0,003340 & 2,772 & 0,00557 \\
Herbáceas & $-0,054179$ & 0,010985 & $-4,932$ & $8,14 \mathrm{e}-07$ \\
Dureza*Sitio & $-0,218702$ & 0,138493 & $-1,579$ & 0,11430 \\
Dureza*Micrositio & $-0,262962$ & 0,093495 & $-2,813$ & 0,00491 \\
Dosel*Sitio & $-0,007120$ & 0,004741 & $-1,502$ & 0,13314 \\
Herbáceas*Sitio & 0,016830 & 0,006167 & 2,729 & 0,00635 \\
Herbáceas*Micrositio & 0,021016 & 0,005035 & 4,174 & $3,00 \mathrm{e}-05$ \\
\hline
\end{tabular}

fue baja en los tres micrositios, aumentando hasta valores de dureza de aproximadamente $1,5 \mathrm{~kg} \mathrm{~cm}^{-2}$, permaneciendo relativamente constante en los claros y vías de saca, $\mathrm{y}$ disminuyendo considerablemente en los caminos a partir de valores de dureza de aproximadamente $2,3 \mathrm{~kg} \mathrm{~cm}^{-2}$. El efecto del dosel tendió a depender del sitio: a bajos valores de cobertura de dosel $(<25 \%)$ la densidad de plantas fue mayor en el sitio 1, a partir de coberturas del $30 \%$ la densidad de plantas fue mayor en el sitio 2, para igualarse a coberturas mayores al $70 \%$. Por último, el efecto de la cobertura herbácea dependió del micrositio y del si- tio (cuadro 6 y figura 4). En suelos con poca cobertura de plantas herbáceas la densidad fue baja, aumentando en los tres micrositios hasta valores de cobertura del orden del 10-15 \%. A partir de esos valores, la densidad de plantas disminuyó en las vías y los claros pero aumentó linealmente en los caminos. La densidad de plantas aumentó en el sitio 1 hasta valores de 10-15\%, disminuyendo gradualmente a medida que aumentaba la cobertura de herbáceas. En el sitio 2 el cambio en la tendencia ocurrió a mayores valores de cobertura de herbáceas (35-40\%) (cuadro 6 y figura 4). 


\section{DISCUSIÓN}

Resumiendo las características de los tres micrositios estudiados, se puede decir que los caminos principales tuvieron los suelos más compactados, las vías de saca los doseles más cerrados y los claros de extracción los suelos con mayor cobertura de herbáceas. En cuanto a la cobertura del dosel, llama la atención que el sitio con menor tiempo de recuperación (sitio 1) posea en promedio valores de cobertura de dosel mayores. Una posible explicación es que en dicho sitio el bosque fue explotado con menor intensidad, o también que los árboles talados fueron de menor tamaño. No se dispone de información en cuanto al número de árboles extraídos por superficie (intensidad de extracción), pero sí se sabe que el diámetro promedio de los troncos talados (diámetros a la altura de la base) fue menor en el sitio con menor tiempo de recuperación ${ }^{1}$ (sitio 1: 32,7 cm \pm $6,5 \mathrm{~cm}$; sitio $2: 35,6 \mathrm{~cm} \pm 9,5 \mathrm{~cm}$; promedio $\pm \mathrm{DE} ; t=2,32$; $P=0.021)$. Esta diferencia de $2,9 \mathrm{~cm}$ podría significar diferencias importantes en las edades de los árboles talados (y, por lo tanto, en el tamaño de sus copas), ya que la especie principalmente explotada (Schinopsis lorentzii) es de lento crecimiento (3-4 mm por año, Araujo et al. 2007).

En función de las características físicas y ambientales de los micrositios, se podría esperar que la composición de especies en los caminos y los claros (micrositos abiertos con mayor porcentaje de luz incidente) sea diferente de la composición en las vías de saca (más cerrados y con menor incidencia de luz), tal como fue sugerido por varios autores (Páez y Marco 2000, Nabe-Nielsen et al. 1997). Sin embargo, en las parcelas estudiadas los tres micrositios (de ambos sitios) estuvieron dominados por las mismas especies: Acacia praecox y Celtis pallida. Más aún, estas especies son las mismas que dominan el ensamble de renovales en los bosques secundarios del área de estudio, en donde hay mayores niveles de cobertura de dosel y, por lo tanto, de sombra (Tálamo 2006). Dado que el tiempo de abandono desde la explotación forestal de estos sitios (1-2 años) fue similar al de los estudiados por Nabe-Nielsen et al. (1997), la diferencia encontrada podría deberse a los distintos niveles de precipitaciones, ya que el bosque estudiado en este trabajo es subtropical semiárido, con lluvias que rondan los 700 $\mathrm{mm}$ anuales, mientras que el bosque estudiado en Bolivia es tropical, con mayores precipitaciones $(1.500 \mathrm{~mm}$ anuales, Nabe-Nielsen et al. 2007). Esta diferencia en el régimen pluvial y la diferente composición de especies de ambos bosques, con tasas de crecimiento diferentes, podrían explicar por qué en el mismo período de tiempo ocurrieron distintos patrones de regeneración en ambos bosques. Por otro lado, los resultados tampoco se corresponden con lo encontrado por Páez y Marco (2000) para un bosque similar al de este estudio (Chaco árido versus Chaco semiárido) con muchas especies en común. Esta diferencia sí podría deberse a que, en un bosque con especies de maderas du-

${ }^{1}$ A Tálamo. Datos no publicados. ras y de bajas tasas de crecimiento, un período de 1-2 años resulte poco tiempo para que los ensambles presentes en micrositios con diferente cobertura de dosel se diferencien en su composición específica. Esta hipótesis debería ser puesta a prueba en futuros estudios, incorporando un gradiente de sitios con diferente tiempo de recuperación desde el abandono del aprovechamiento forestal.

La densidad de plantas leñosas dependió del micrositio considerado y del tiempo de recuperación y de su interacción con las co-variables estudiadas. La menor densidad de plantas leñosas encontrada en los caminos principales podría deberse a la mayor dureza del suelo registrada en las parcelas de ambos sitios. Esta compactación podría comprometer el reclutamiento de algunas especies en el área de estudio, tal como ya ha sido documentado en otros bosques (Pinard et al. 1996, Guariguata y Dupuy 1997). En caminos de extracción abandonados en distintos tipos de bosques, muchos atributos comunitarios y estructurales son diferentes a los del bosque adyacente, y una de las causas propuestas es la dureza de los suelos (Guariguata y Dupuy 1997, Pinard et al. 2000, Nabe-Nielsen et al. 2007). Además de la compactación, las condiciones microclimáticas generadas por la construcción del camino (altas temperatura en el suelo, bajos contenidos de nutrientes y de humedad) podrían también limitar el establecimiento y la supervivencia de renovales (Pinard et al. 1996, Burton y Bazzas 1991), influyendo en la densidad final de plantas leñosas. Esto podría también explicar la baja cobertura de plantas herbáceas dicotiledóneas de los caminos principales en los dos sitios estudiados. Coincidiendo con Ampoorter et al. (2011), en este trabajo se observó que el efecto de la dureza del suelo no siempre es negativo, ya que en las vías de saca se observa un leve aumento de la densidad a medida que aumenta la dureza del suelo, mientras que en los caminos hay una importante disminución de la densidad asociada a la dureza del suelo. El efecto de la dureza depende del tipo de suelo (Ampoorter et al. 2011) y esa podría ser una explicación a los resultados encontrados en este estudio, ya que algunas de las parcelas estudiadas se situaban en suelos un poco más arenosos que otros. Por su parte, los efectos de la cobertura del dosel y de la cobertura de plantas herbáceas en los caminos principales resultaron opuestos al de la dureza del suelo. En estos micrositios más afectados (con suelos más duros, doseles más abiertos y suelos menos cubiertos), la sombra generada por el dosel y la cobertura de las plantas herbáceas jugarían un rol positivo, mientras que, lo contrario, ocurriría en micrositios menos afectados (con suelos más blandos y con mayor cobertura). Esto podría estar relacionado con lo predicho por la hipótesis del gradiente de estrés (SGH), en donde se espera que el efecto de la facilitación sea más importante a mayores valores de estrés y que la competencia sea la interacción predominante en ambientes con menos estrés (Anthelme y Michalet 2009, Malkinson y Tielbörger 2010).

La ausencia en este estudio de un tratamiento control de micrositio (parcelas en la matriz circundante) no permi- 
tió evaluar si la regeneración en los micrositios es diferente a la de la matriz y, por lo tanto, no es posible saber si el aprovechamiento forestal está impactando de alguna manera sobre la regeneración de las especies de valor forestal. Sin embargo, se puede realizar una comparación indirecta ya que se dispone de datos de regeneración de Schinopsis lorentzii y Aspidosperma quebracho-blanco obtenidos en un muestreo simultáneo (12 parcelas de 2 x $50 \mathrm{~m}$ en el sitio 1 y el sitio 2; Tálamo 2006). Ambas especies presentaron una regeneración casi nula en los micrositios del sitio con menor tiempo de recuperación (sitio 1). En el sitio 2 estuvieron presentes en casi todos los micrositios, pero en bajas densidades y con baja constancia (i.e., baja proporción de parcelas con individuos). Los valores de regeneración de A. quebracho-blanco en los micrositios fueron similares a los encontrados en la matriz circundante $(0,04$ individuos $\mathrm{m}^{-2}$ ), mientras que para $S$. lorentzii los valores de regeneración de los micrositios $(0,05-0,06$ individuos $\mathrm{m}^{-2}$; cuadro 4) fueron superiores a los de la matriz $(0,01$ individuos $\mathrm{m}^{-2}$ ) (Tálamo 2006). Por lo tanto, una posible interpretación es que la generación de diferentes micrositios como consecuencia del aprovechamiento forestal no sería algo negativo para la regeneración de las especies de interés forestal en este ambiente. De todos modos, estas conclusiones deben ser interpretadas con cautela ya que la comparación comprende datos obtenidos con tamaños de parcela muy diferentes $\left(1 \mathrm{~m}^{2}\right.$ versus $\left.100 \mathrm{~m}^{2}\right)$ y las estimaciones para los micrositios presentaron una alta variabilidad (cuadro 4) debido a que las especies de interés forestal estuvieron representadas en una baja proporción de las parcelas estudiadas.

En conclusión, a diferencia de lo planteado en la primera predicción relacionada con la composición específica y los niveles de cobertura de dosel (sombra), la composición de especies y la riqueza de plantas leñosas fue similar en los tres micrositios (caminos, vías de saca y claros de extracción). Probablemente, el tiempo transcurrido desde al abandono del aprovechamiento forestal no fue suficiente como para permitir una diferenciación en la composición. En relación a la segunda predicción, la densidad de plantas leñosas fue diferente entre micrositios debido a la interacción con las variables bióticas y abióticas que caracterizaron a los mismos. La regeneración de las especies de valor forestal fue baja en comparación a la de otras especies, pero A. quebracho-blanco tuvo una densidad similar y $S$. lorentzii una densidad mayor en comparación a la regeneración observada por Tálamo (2006) en la matriz circundante. Se propone, con cautela, que los micrositios generados por el aprovechamiento no estarían afectando negativamente a la regeneración de las dos especies de mayor valor forestal de este bosque.

\section{AGRADECIMIENTOS}

Este trabajo fue posible gracias al apoyo de CONICET (Beca Doctoral Interna) y Rufford Small Grant Foundation por los subsidios otorgados. Agradecemos profundamente a Verónica Quiroga, Enrique Derlindati y Jorge Lucema por el invaluable apoyo brindado durante la toma de datos en el campo. Tres revisores anónimos realizaron valiosos comentarios que mejoraron versiones anteriores del manuscrito. El trabajo está dedicado a Sandra Caziani, quien no pudo ver la versión final de este y otros manuscritos en los que participó activamente como autora.

\section{REFERENCIAS}

Ampoorter A, P De Frenne, M Hermy, K Verheyen. 2011. Effects of soil compaction on growth and survival of tree saplings: a meta-analysis. Basic and Applied Ecology 12: 394-402.

Anthelme F, R Michalet. 2009. Grass-to-tree facilitation in an arid grazed environment (Air mountains, Sahara). Basic and Applied Ecology 10: 437-446.

Araujo P, M Juárez de Galíndez, M Iturre. 2007. Crecimiento de las especies principales de un bosque en regeneración del Chaco santiagueño. Quebracho 14: 36-46.

Brassiolo M, A Tasso, M Abt, G Merletti. 2001. Diagnóstico socioeconómico y de uso del suelo en la zona de amortiguamiento del Parque Nacional Copo. Informe Técnico. Santiago del Estero, Argentina. APN-GEF-BIRF. 90 p.

Burnham KP, DR Anderson. 2002. Model selection and multimodel inference: a practical information-theoretic approach. 2nd ed. Fort Collins, USA. Springer-Verlag. 488 p.

Burton PJ, FA Bazzaz. 1991. Tree seedling emergence on interactive temperature and moisture gradients and in patches of old-field vegetation. American Journal of Botany 78: 131-151.

Colwell RK. 2011. Estimates: Statistical Estimation of Species Richness and Shared Species from Samples. Version 8.2.0. User's guide and application. Consultado 24 ene. 2012. Disponible en http://purl.oclc.org/estimates/

Colwell RK, A Chao, JN Gotelli, SY Lin, CX Mao, RL Chazdon, JT Longino. 2012. Models and estimators linking individual-based and sample-based rarefaction, extrapolation and comparison of assemblages. Journal of Plant Ecology 5: 3-21.

Davis MA, KJ Wrage, PB Reich. 1998. Competition between tree seedlings and herbaceous vegetation: support for a theory of resource supply and demand. Journal of Ecology 86: 652-661.

Feinsinger P. 2004. El diseño de estudios de campo para la conservación de la biodiversidad. Santa Cruz de la Sierra, Bolivia. Editorial FAN. 243 p.

Fredericksen TS, W Pariona. 2002. Effect of skidder disturbance on commercial tree regeneration in logging gaps in a Bolivian tropical forest. Forest Ecology and Management 171: 223-230.

Grulke M, M Brassiolo, F Díaz Lanes, K Obst, G Ortíz, G Soto, J Michela. 2007. Manual para el manejo forestal sustentable de los bosques nativos de la Provincia del Chaco. Chaco, Argentina. Ministerio de la Producción del Gobierno de la Provincia del Chaco. 176 p.

Guariguata MR, JM Dupuy. 1997. Forest regeneration in abandoned logging roads in Lowland Costa Rica. Biotropica 29: 15-28.

Malkinson D, K Tielbörger. 2010. What does the stress-gradient 
hypothesis predict? Resolving the discrepancies. Oikos 119: 1546-1552.

Mostacedo BC, TS Fredericksen. 2001. Regeneración y Silvicultura de Bosques Tropicales en Bolivia. Santa Cruz de la Sierra, Bolivia. Proyecto de Manejo Forestal Sostenible (BOLFOR). $221 \mathrm{p}$.

Nabe-Nielsen J, W Severiche, T Fredericksen, LI Nabe-Nielsen. 2007. Timber tree regeneration along abandoned logging roads in a tropical Bolivian forest. New Forests 34: 31-40.

Nicotra AB, RL Chazdon, SV Iriarte. 1999. Spatial heterogeneity of light and woody seedling regeneration in tropical wet forests. Ecology 80: 1908-1926.

Páez SA, DE Marco. 2000. Seedling habitat structure in dry Chaco forest (Argentina). Journal of Arid Environment 46: 5768.

Park A., MJ Justiniano, TS Fredericksen. 2005. Natural regeneration and environmental relationships of tree species in logging gaps in a Bolivian tropical forest. Forest Ecology and Management 217: 147-157.

Peña-Claros M, EM Peters, MJ Justiniano, F Bongers, GM Blate, TS Fredericksen, FE Putz. 2008. Regeneration of commercial tree species following silvicultural treatments in a moist tropical forest. Forest Ecology and Management 255: 1283-1293.
Pinard M, B Howlett, D Davidson. 1996. Site conditions limits pioneer tree recruitment after logging of dipterocarp forest in Sabah, Malaysia. Biotropica 28: 2-12.

Pinard MA, MG Barker, J Tay. 2000. Soil disturbance and postlogging forest recovery on bulldozer path in Sabah, Malaysia. Forest Ecology and Management 130: 213-225.

Quinn G, M Keough. 2002. Experimental design and data analysis for biologists. New York, USA. Cambridge University Press. 537 p.

R Development Core Team. 2010. R: A Language and Environment for Statistical Computing. Disponible en: http:// www.r-project.org/

Tálamo A. 2006. Biodiversidad de plantas leñosas y disturbios humanos en el bosque chaqueño semiárido: efectos del aprovechamiento forestal. Tesis Doctoral. Buenos Aires, Argentina. Facultad de Cs. Exactas y Naturales, Universidad de Buenos Aires. 110 p.

Toledo-Aceves T, S Purata-Velarde, CM Peters. 2009. Regeneration of commercial tree species in a logged forest in the Selva Maya, Mexico. Forest Ecology and Management 258: 2481-2489.

Zak MR, M Cabido, JG Hodgson. 2004. Do subtropical seasonal forests in the Gran Chaco, Argentina, have a future? Biological Conservation 129: 589-598.

Recibido: 22.02.12

Aceptado: 03.09.12 